
JNM
J Neurogastroenterol Motil, Vol. 22 No. 1 January, 2016
pISSN: 2093-0879 elSSN: 2093-0887
http://dx.doi.org/10.5056/jnm15118

\title{
Topological Alterations of the Intrinsic Brain Network in Patients with Functional Dyspepsia
}

\author{
Jiaofen Nan, ${ }^{1}$ Li Zhang, ${ }^{2}$ Fubao Zhu, ${ }^{1}$ Xiaorui Tian, ${ }^{3}$ Qian Zheng, ${ }^{1}$ Karen M von Deneen, ${ }^{4}$ Jixin Liu, ${ }^{4 *}$ and Ming Zhang ${ }^{2 *}$ \\ ${ }^{I}$ Zhengzhou University of Light Industry, Zhengzhou, China; ${ }^{2}$ Department of Medical Imaging; First Affiliated Hospital of Xi'an Jiaotong \\ University, Xi'an, China; ${ }^{3}$ The Fifth Affiliated Hospital of Zhengzhou University, Zhengzhou, China; and ${ }^{4}$ School of Life Science and Technology, \\ Xidian University, Xi'an, China
}

\section{Background/Aims}

Previous studies reported that integrated information in the brain ultimately determines the subjective experience of patients with chronic pain, but how the information is integrated in the brain connectome of functional dyspepsia (FD) patients remains largely unclear. The study aimed to quantify the topological changes of the brain network in FD patients.

\section{Methods}

Small-world properties, network efficiency and nodal centrality were utilized to measure the changes in topological architecture in 25 FD patients and 25 healthy controls based on functional magnetic resonance imaging. Pearson's correlation assessed the relationship of each topological property with clinical symptoms.

\section{Results}

FD patients showed an increase of clustering coefficients and local efficiency relative to controls from the perspective of a whole network as well as elevated nodal centrality in the right orbital part of the inferior frontal gyrus, left anterior cingulate gyrus and left hippocampus, and decreased nodal centrality in the right posterior cingulate gyrus, left cuneus, right putamen, left middle occipital gyrus and right inferior occipital gyrus. Moreover, the centrality in the anterior cingulate gyrus was significantly associated with symptom severity and duration in FD patients. Nevertheless, the inclusion of anxiety and depression scores as covariates erased the group differences in nodal centralities in the orbital part of the inferior frontal gyrus and hippocampus.

\section{Conclusions}

The results suggest topological disruption of the functional brain networks in FD patients, presumably in response to disturbances of sensory information integrated with emotion, memory, pain modulation, and selective attention in patients.

(J Neurogastroenterol Motil 2016;22:118-128)

\section{Key Words}

Brain; Connectome; Dyspepsia; Functional magnetic resonance imaging

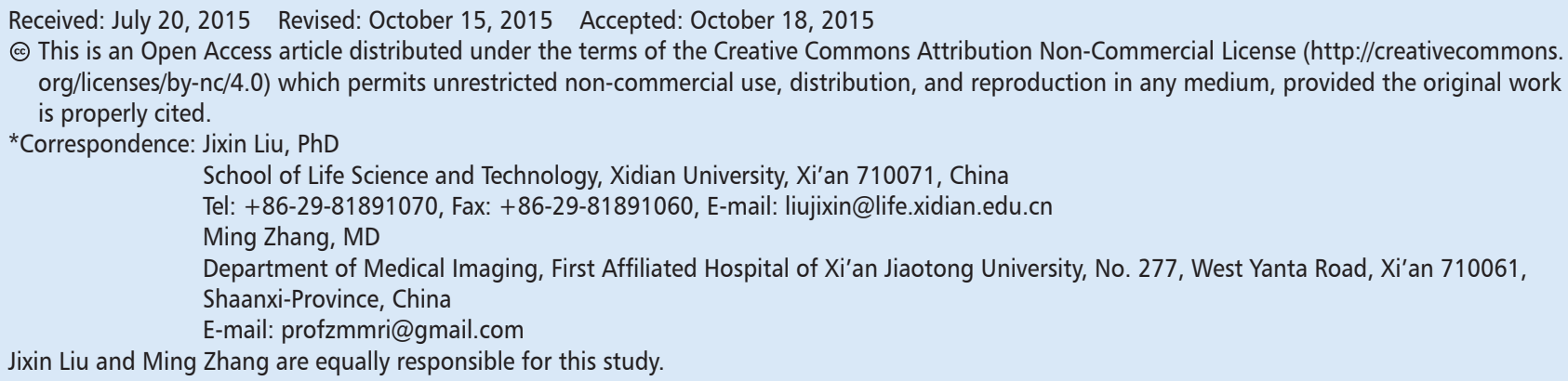

(a) This is an Open Access article distributed under the terms of the Creative Commons Attribution Non-Commercial License (http://creativecommons. org/licenses/by-nc/4.0) which permits unrestricted non-commercial use, distribution, and reproduction in any medium, provided the original work is properly cited.

*Correspondence: Jixin Liu, PhD

School of Life Science and Technology, Xidian University, Xi'an 710071, China

Tel: +86-29-81891070, Fax: +86-29-81891060, E-mail: liujixin@life.xidian.edu.cn

Ming Zhang, MD

Department of Medical Imaging, First Affiliated Hospital of Xi'an Jiaotong University, No. 277, West Yanta Road, Xi'an 710061,

Shaanxi-Province, China

E-mail: profzmmri@gmail.com

Jixin Liu and Ming Zhang are equally responsible for this study. 


\section{Introduction}

Functional dyspepsia (FD), one of the most common types of functional gastrointestinal disorders (FGID), is defined by a symptom criteria based on Rome III. ${ }^{1}$ It is characterized by the presence of recurrent symptoms of early satiety, fullness, nausea and pain referred to the upper abdomen. ${ }^{2}$ Although there are no organic lesions, FD could cause a heavy burden for patients and their families because of repeated gastric symptoms. Therefore, it has realistic significance to study pathophysiology underlying symptom generation. Currently, the central nervous system is widely accepted to be important in symptom generation of FD. There is an increasing consensus that multiple factors encompassing emotion, cognition, reward, past memories of discomfort experiences, and salience appraisal are integrated with sensory information in the human brain, and the integrated information ultimately determines the subjective experience of patients with chronic pain, ${ }^{3-5}$ such as FD and irritable bowel syndrome (IBS). However, it remains unclear how the information is integrated in the brain architecture in FD patients.

Recent studies using modern brain mapping techniques suggested that FD is related to the widespread abnormalities in local brain regions, such as the anterior cingulate gyrus (ACG), insula, middle cingulate gyrus, thalamus, prefrontal cortex, and somatosensory cortex. ${ }^{6-10}$ Moreover, alterations in brain links have been identified in FD patients, such as ACG-insula connectivity, insula-frontal connectivity, ACG-frontal connectivity, thalamus-sensorimotor connectivity and so on. ${ }^{9,11}$ The above studies provided regional disturbances and changes in links between region pairs in FD patients, improving the understanding of the pathological disorders of FD. However, they ignored the complexity of the regional interactions as an entire network. In contrast, topological analyses characterized the brain network as a whole by detecting the patterns of local and global circuits. ${ }^{12}$ Currently, very little is known regarding whether and how FD disrupts the topological organization of the intrinsic brain networks.

Complex network analysis (stems from the graph theory of mathematics), provides an approach for quantifying topologies of the brain network involving multiple aspects of brain connectivity. ${ }^{12}$ As a complex system, a brain network is neither ordered nor random and it is functionally organized in small-world architecture with low cost, high efficiency and high modularity. ${ }^{13}$ Many brain diseases (schizophrenia, ${ }^{14}$ post-traumatic stress disorder, ${ }^{15}$ major depressive disorder, ${ }^{16}$ and epilepsy ${ }^{17,18}$ ) are linked to the disturbed topological organization in the brain network. Imaging studies have documented that FD is a disease associated with whole-brain disorders, ${ }^{11}$ thus we hypothesized that there were alterations in the brain network topology of FD patients. Moreover, the changes may be closely related to the increase in dyspepsia symptoms.

To test this hypothesis, we used resting-state functional MRI to investigate the topological properties of brain networks at local and global levels in FD patients. The topological characteristics were calculated by using a complex network analysis. Based on 25 FD patients and 25 healthy subjects, we investigated topological differences between the two groups and their relationships with clinical symptoms.

\section{Materials and Methods}

This study protocol was approved by the Institutional Review Board of the First Affiliated Hospital of the Medical College in Xi'an Jiaotong University, and written informed consent was obtained from all subjects.

\section{Subjects}

According to the need of the study, the subjects were interviewed and screened by a digestive specialist. After an initial assessment, each patient was re-examined according to the inclusion and exclusion criteria in Rome III for diagnosis of FD. Inclusion criteria were characterized by the presence of recurrent symptoms in the upper abdomen. Exclusion criteria included a history of drug abuse and head trauma with loss of consciousness, esophagitis, erosive gastroduodenal lesions, gastric ulcers, gastric atrophy, mental deficiency, psychiatric and neurological disorders, hepatic, renal, respiratory, and cardiovascular diseases, pregnant or lactating women, and any contraindication to an MRI. A total of 26 patients were recruited in the age range of 20 to 30 years. One FD patient was discarded due to the silent lesions and the other 2 patients received 2 scans for excessive head movement during the first scan (see Data Acquisition and Data Preprocessing below).

A total of 30 healthy controls (HC) were carefully evaluated through a personal questionnaire survey about a history of medication or diseases, and complete physical examinations. No controls had a history of gastrointestinal disorders or other serious diseases. To match the demographic information of the patient group, 25 controls were selected from the healthy subjects screened above.

\section{Clinical Questionnaires}

The severity of dyspepsia symptoms was assessed using a dyspepsia symptom questionnaire, which included four items (early 
satiety, epigastric pain, burning, and abdominal fullness or discomfort) with each item rated on a four-point scale. The larger the dyspepsia symptom score (DSS), the more severe the illness.

The levels of anxiety and depression were rated respectively using the 20-item self-rating anxiety scale (SAS) ${ }^{19}$ and self-rating depression scale (SDS). ${ }^{20}$ The higher the scores for SAS and SDS, the more severe the anxiety and depression.

\section{Data Acquisition}

All subjects underwent a functional MRI scan with a 3 Tesla Signa GE magnetic resonance scanner (GE Healthcare, Milwaukee, WI, USA) at the Department of Medical Imaging, First Affiliated Hospital of Xi'an Jiaotong University, Xi'an, China. To exclude silent lesions, a $\mathrm{T} 1$ structural image was examined for each participant and the parameters were as follows: echo time $=2.26$ milliseconds, repetition time $=1900$ milliseconds, flip angle $=9^{\circ}$, matrix $=256 \times 256$, field of view $=256 \times 256 \mathrm{~mm}$, number of slices $=176$, and voxel size $=1 \mathrm{~mm}^{3}$. The FD patients and healthy subjects without silent lesions underwent a functional MRI scan with a gradient echo planar imaging sequence. The parameters were as follows: echo time $=30$ milliseconds, repetition time $=2$ seconds, flip angle $=90^{\circ}$, matrix $=64 \times 64$, field of view $=240$ $\times 240 \mathrm{~mm}$, in-plane spatial resolution $=3.75 \times 3.75 \mathrm{~mm}^{2}$, slicethickness $=5 \mathrm{~mm}$, number of slices per volume $=30$, and no section gap. During the scan, the participants were asked to neither fall asleep nor think about anything in particular. The functional scan lasted 6 minutes (a total of 180 volumes). To be eligible for the resting-state study, we asked each participant if he or she was awake and relaxed during the scan.

\section{Data Preprocessing}

Data preprocessing was carried out using SPM8 (http://www. fil.ion.ucl.ac.uk/spm/). The steps were as follows: first, the first 10 volumes for each subject were removed to eliminate the effects of magnetization stabilization. Next, the remaining images were corrected for intra-volume time delay and head movement. Because of excessive translational movement $(>1.5 \mathrm{~mm}$ ) or rotation (> $1.5^{\circ}$ ), we rescanned 2 patients. The corrected images were spatially normalized to the Monteal Neurological Institute (MNI) 152 templates and resampled to $3 \times 3 \times 3 \mathrm{~mm}^{3}$ voxels. Next, the normalized images were linearly detrended and temporally bandpass filtered $(0.01-0.08 \mathrm{~Hz})$ to eliminate the scanner drift and physiological noise. Finally, the nuisances were further removed from the resulting images by regressing out head motion parameters, the cerebral spinal fluid signal, and white-matter signal.

\section{Brain Network Construction}

A brain network is composed of brain regions (nodes or vertices in a complex network) and connections (edges or links in a complex network). The whole brain was herein divided into 90 regions of interests according to the automated anatomical labeling (AAL) atlas, ${ }^{21}$ with each ROI representing a node (see Supplementary Table 1). Functional connections are defined by the Pearson correlation coefficient between the mean time series of all pairs of nodes, and reflect interactions of blood oxygen level dependent signals of brain dynamics. ${ }^{12}$ In the study, the residuals of images after data preprocessing were utilized to calculate the cross-correlations between all possible pairs of 90 nodes, forming a $90 \times 90$ matrix of correlation coefficients for each subject.

\section{Brain Network Analysis}

All self-correlations and negative correlations were removed (set to 0 ) from the correlation matrix prior to the brain network analysis. To minimize possible discrepancies and facilitate the statistical analysis, the individual brain network (expressed in the correlation matrix) was converted to a binarized network (expressed in an adjacent matrix $\mathrm{A}=\left[\mathrm{a}_{\mathrm{ij}}\right], \mathrm{a}_{\mathrm{ij}}=1$ or 0 ) by determining a sparsity threshold $S$ (a percentage that equals the ratio of the number of connections in the resultant network divided by the maximum possible number of connections). The steps included: (1) sorting the correlation coefficients from largest to smallest and (2) retaining the connections ranked in the top percent. This method normalized all cortical networks so they have the same number of connections. To not arbitrarily set the threshold, a wide range of S $(0.15<S<$ 0.3 with an interval of 0.01 ) was applied to the correlation matrix according to the method mentioned above to transform it into the adjacent matrix. The range of $S$ could assure small-worldness and sparse properties as well as minimize the number of spurious edges in the cortical networks. In the current study, $S=0.16$ is the smallest threshold used to assure the full connectedness of individual network.

In this study, we investigated the topologies of all binary networks at each threshold. The topological attributes included smallworld properties (clustering coefficient, shortest path length, normalized clustering coefficient, and normalized shortest path length), network efficiency (local efficiency and global efficiency) and nodal metrics (degree, nodal efficiency, and betweenness). Based on a binarized cortical network $G$ with $n$ vertices, the detailed definitions of the above metrics were as follows, and their meanings are summarized in Supplementary Table 2. 


\section{Clustering coefficient and normalized clustering coefficient}

The clustering coefficient of the network is the mean of the nodes' clustering coefficients, as shown in equation (1).

$$
C=\frac{1}{n} \sum_{i=1}^{n} C_{i}
$$

In equation (1), $\mathrm{n}$ is the number of brain nodes in the cortical network $\mathrm{G}$, where $C_{i}$ represents the clustering coefficient of the ith node and $C_{i}=\frac{E_{i}}{D_{i}\left(D_{i}-1\right) / 2} \cdot D_{i}$ is the number of vertices connected to the ith node (that is, the number of neighbors of the ith node) and $E_{i}$ is the number of actual edges between the neighbors of the ith node. We used $C_{\text {real }}$ and $C_{\text {rand }}$ to denote the clustering coefficient of the real brain network and random network with the same number of nodes, and the normalized clustering coefficient can be defined as follows: $\gamma=C_{\text {real }} / C_{\text {rand }}$.

\section{Shortest path length and normalized shortest path length}

The shortest path length of the network is the mean of the shortest path lengths between all possible pairs of nodes, as shown in equation (2).

$$
L=\frac{1}{n(n-1)} \sum_{i \neq j \in G} L_{i j}
$$

In equation (2), $L_{i j}$ represents the minimum of the edge number for all paths of node $i$ connected to node $j$. We used $L_{\text {real }}$ and $L_{\text {rand }}$ to denote the shortest path length of the real brain network and random network with the same number of nodes, and the normalized shortest path length can be defined as follows: $\lambda=L_{\text {real }} / L_{\text {rand }}$.

\section{Small-world network}

If a network has a high clustering coefficient and short path length, we considered it as a small-world network. Using the random network as a benchmark, a "small-world" brain network should simultaneously satisfy $\mathrm{C}_{\text {real }} \gg C_{\text {rand }}$ (namely, $\gamma=\mathrm{C}_{\text {real }} / C_{\text {rand }}>1$ ) and $L_{\text {real }} \approx L_{\text {rand }}$ (namely, $\left.\lambda=L_{\text {real }} / L_{\text {rand }} \approx 1\right)$.

\section{Network efficiency}

Efficiency describes the network architecture from the perspective of information flow, measuring the ability of information transfer of a given network. ${ }^{22}$ Global efficiency is calculated based on the shortest path lengths as follows:

$$
E_{\text {global }}=\frac{1}{n(n-1)} \sum_{i \neq j \in G} \frac{1}{L_{i j}}
$$

The local efficiency is defined as

$$
E_{\text {local }}=\frac{1}{n} \sum_{i=1}^{n} E_{\text {global }}(i)
$$

In equation (4), $E_{\text {global }}$ (i) is the global efficiency of the subgraph composed of all neighbors of the ith node.

\section{Nodal centrality}

Nodal centrality can be used to measure the functional interaction patterns within the brain connectome. Different aspects of brain connectivity can be captured by various centrality indices. ${ }^{23}$ To quantify the contribution of the brain nodes to different aspects of connectivity in a cortical network, we calculated 3 nodal indices: degree centrality, betweenness, and nodal efficiency. They can be divided into local (degree), mesoscale (nodal efficiency), and global centralities (betweenness).

Degree centrality of a node is the number of neighbors of the node. ${ }^{23}$ Given the binarized matrix $A=\left[a_{i j}\right]$, the degree centrality can be calculated in $D_{i}=\sum_{j=1}^{n} a_{i j}$. It is a direct centrality measure reflecting the most local centrality of the network.

The nodal efficiency is computed as

$$
E_{\text {nodal }}(i)=\frac{1}{n-1} \sum_{j \neq i \in \mathrm{G}} \frac{1}{L_{i j}},
$$

where $L_{i j}$ represents the shortest path length of node $i$ connected to node $j$. This measure quantifies the ability of information transfer from a given node to the other nodes in the cortical network. $^{22}$

The betweenness centrality of a brain node denotes the number of shortest paths between all possible pairs for the rest of the nodes through the given node, ${ }^{24}$ which is computed in

$$
B_{i}=\sum_{j \neq k \neq i \in G} \frac{\delta_{j k}(i)}{\delta_{j k}},
$$

where $\delta_{j k}$ (i) is the number of shortest paths from the $j$ th node to the $k$ th node that passes through the ith node. $\delta_{j k}$ is the number of all shortest paths from the $j$ th node to the $k$ th node. This measure characterizes the influence of a given region over information flow within a cortical network, which captures the nodal centrality at a global level.

\section{Statistical Methods}

The above properties were computed based on the individual level. We examined whether there were altered topological properties in functional brain networks in FD patients using two-sample 
random permutation tests as follows. We considered a significant change in FD patients for each property using the following approach. First, we calculated the difference between FD patients and the $\mathrm{HC}$ group for one property of the brain networks using a twosample $t$ test method. Next, the property values in the patient group and control group were randomly permutated 1000 times and the difference was re-computed after each permutation. Then, $1000 \mathrm{P}$ values could form a null distribution. If the real difference before permutation reached the level 0.05 of the null distribution, the result was considered to be significant at $P<0.05$ after the permutation test. In addition, the correlation analysis was conducted using the Pearson correlation.

\section{Results}

The demographic and clinical characteristics of FD patients and controls are summarized in Table 1 . There were no significant group differences in terms of gender, age, weight, and height $(P>$ 0.05). Moreover, none of the controls went beyond the normal range of anxiety and depression, but 4 patients showed mild anxiety (SAS $>50$ ) and 5 patients showed mild depression (SDS > 53).

\section{Altered "Small-world" Properties}

In the range of the threshold $(0.15<S<0.3)$, FD patients and $\mathrm{HC}$ showed typical properties of a small-world network. That is to say, compared with degree-matched random networks, the cortical network exhibited much larger clustering coefficients and approximately the shortest path length (Fig. 1). Compared with the control group, the FD group had a significantly higher clustering coefficient across the sparsity thresholds of 0.15 to 0.3 , as shown in Figure 2A ( $\mathrm{T}=4.252, P=9.71 \mathrm{e}^{-5}$ at $\left.S=0.16\right)$. No groupdifference was found in the shortest path length (Fig. 2B). In FD patients, the correlation analysis did not reveal a linear relationship between "small-world" metrics and dyspepsia severity.

Table 1. Clinical Information of Subjects

\begin{tabular}{lcccc}
\hline \multicolumn{1}{c}{ Items } & FD group $(\mathrm{n}=25)$ & HC group $(\mathrm{n}=25)$ & $\mathrm{T}^{\mathrm{n}}$ & $P_{\text {-value }}$ \\
\hline Gender (female/male) & $19 / 6$ & $14 / 11$ & 1.496 & 0.141 \\
Age (yr) & $21.46 \pm 4.76$ & $22.08 \pm 1.08$ & -0.634 & 0.529 \\
Weight (kg) & $48.54 \pm 11.74$ & $51.88 \pm 7.81$ & -1.192 & 0.239 \\
Height (cm) & $154.5 \pm 32.37$ & $162.44 \pm 6.99$ & -1.199 & - \\
Duration (mo) & $37.88 \pm 31.27$ & - & - & - \\
DSS & $3.85 \pm 1.43$ & $35.9 \pm 6.39$ & 2.01 & 0.050 \\
SAS & $41.15 \pm 11.49$ & $35.65 \pm 7.66$ & 2.03 & 0.048 \\
SDS & $41.68 \pm 12.82$ & & - \\
\hline
\end{tabular}

${ }^{\mathrm{a}}$ Independent sample T-test.

FD, functional dyspepsia; HC, healthy controls; DSS, dyspepsia symptom score; SAS, self-rating anxiety scale; SDS, self-rating depression scale.

Data were shown as mean $\pm \mathrm{SD}$.
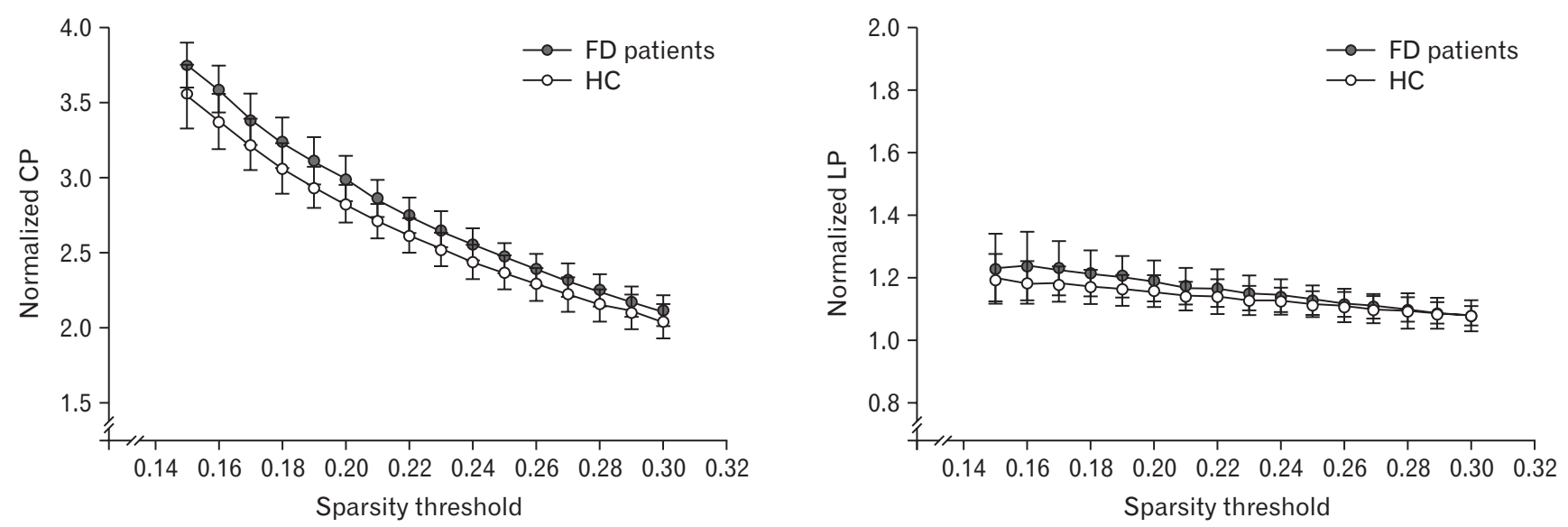

Figure 1. Normalized clustering coefficient and normalized shortest path length in functional dyspepsia (FD) patients compared with healthy controls $(\mathrm{HC})$ in the wide range of the threshold $(0.15<S<0.3)$. CP, clustering coefficient; LP, shortest path length. 
A

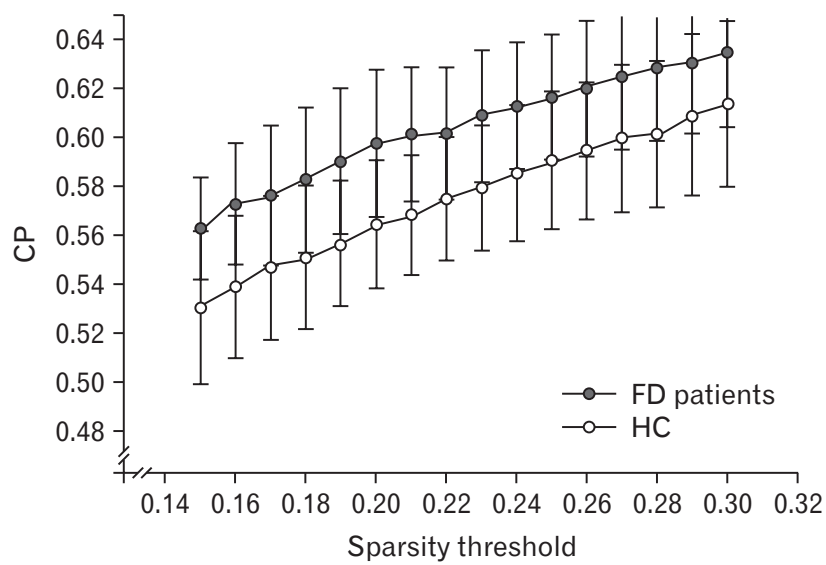

C

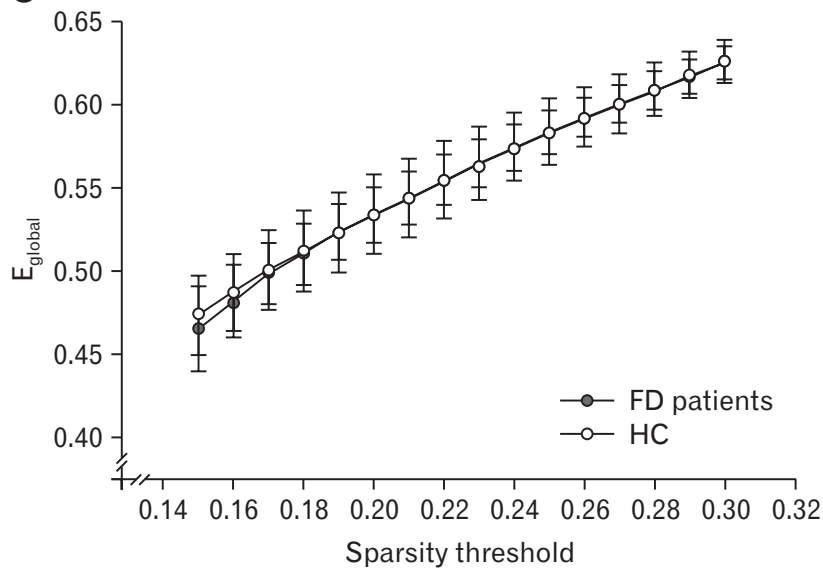

B

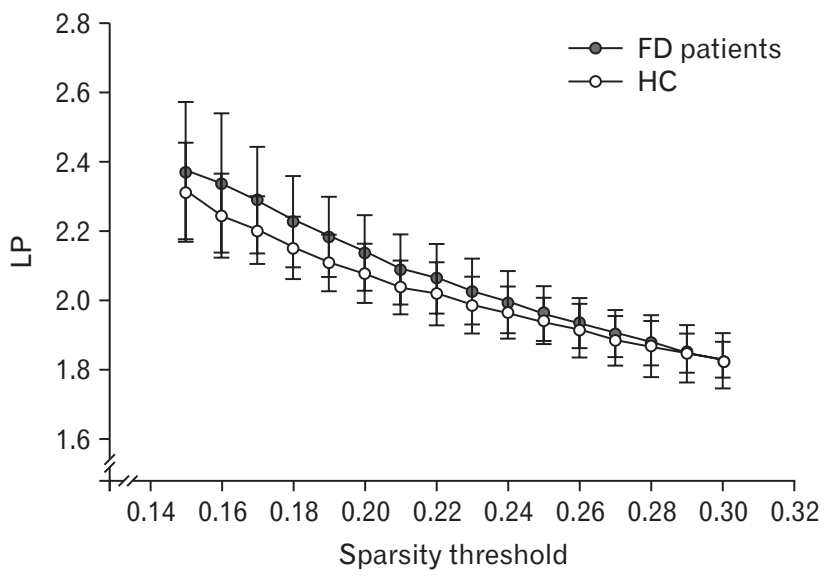

D

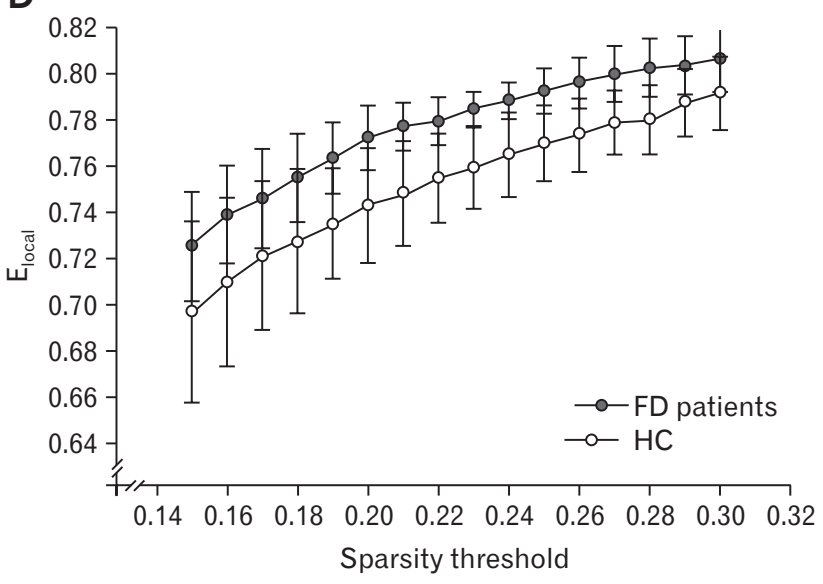

Figure 2. Brain network properties vary with the sparsity threshold in functional dyspepsia (FD) patients and healthy controls (HC). CP, clustering coefficient (A); LP, shortest path length (B); $\mathrm{E}_{\text {global }}$, global efficiency $(\mathrm{C})$; $\mathrm{E}_{\text {local }}$, local efficiency (D).

\section{Altered Network Efficiency}

Statistical analyses showed no significant differences for global efficiency (Fig. 2C), but had a markedly higher local efficiency in patients relative to controls over the entire range of the threshold $(0.15<S<0.3)$ as shown in Figure 2D $\left(\mathrm{T}=4.934, P=1.01 \mathrm{e}^{-5}\right.$ at $S=0.16)$. No significant correlations were found between network efficiency and DSS in FD patients.

\section{Nodal Metrics}

When comparing FD patients with controls, results were notably different for those obtained from degree centrality, betweenness, and local efficiency (Fig. 3). Since the results for the group comparisons were highly similar across the sparsity thresholds of 0.15 to 0.3 , we reported the results at a fully connected threshold ( $S$ $=0.16)$ as a representation. We identified the brain areas that had significant group differences at one of the three centrality indices, as shown in Figure 3A. The degree centrality was involved in higher values in the right orbital part of the inferior frontal gyrus (ORBinf) and lower values in the right putamen in FD patients (Fig. 3B). The nodal efficiency showed an increase in the right ORBinf and a reduction in the left cuneus and right putamen (Fig. 3C). The betweenness centrality exhibited a regional increase encompassing the right ORBinf, left ACG and left hippocampus, and regional decrease composed of the right posterior cingulate gyrus (PCG), left cuneus, left middle occipital gyrus (MOG) and right inferior occipital gyrus (IOG) (Fig. 3D).

Importantly, the degree centrality and nodal efficiency in the ACG were positively correlated to the severity of FD (Fig. 4A and 4B). Moreover, nodal efficiency in the ACG was also positively associated with duration in patients (Fig. 4C). No significant correlations were found between the other centrality metrics and clinical 
A
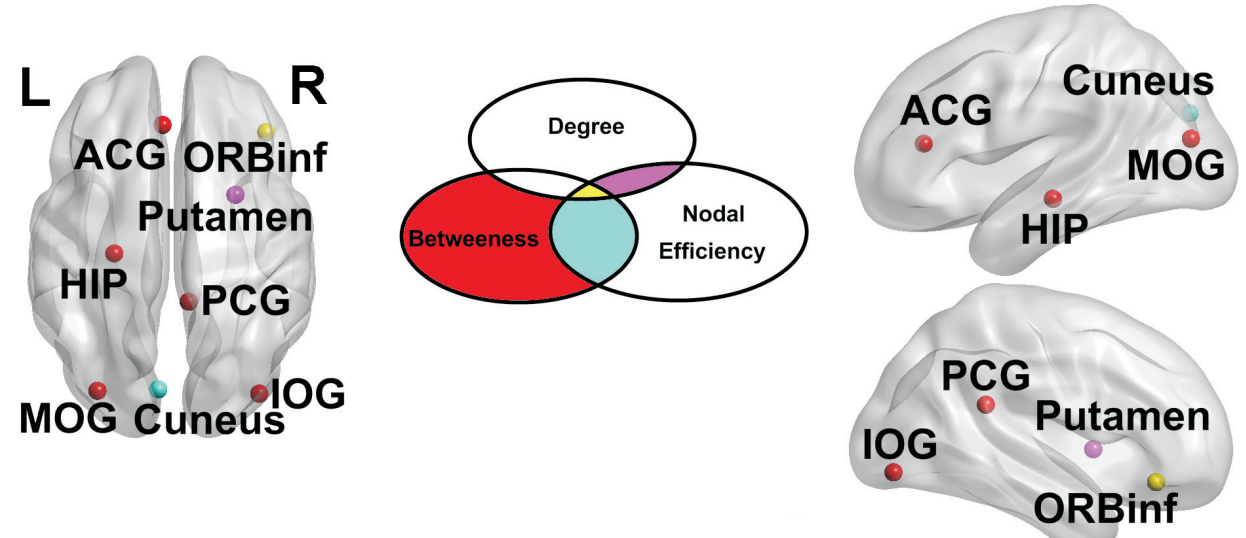

B

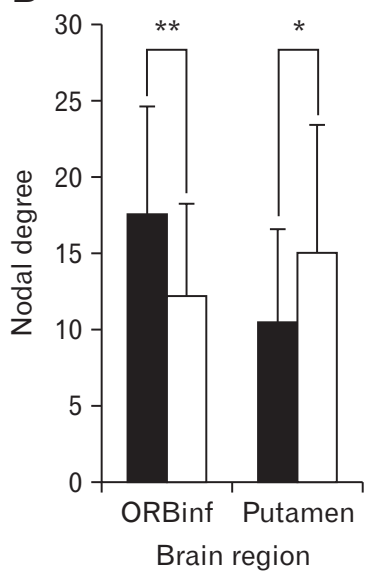

C
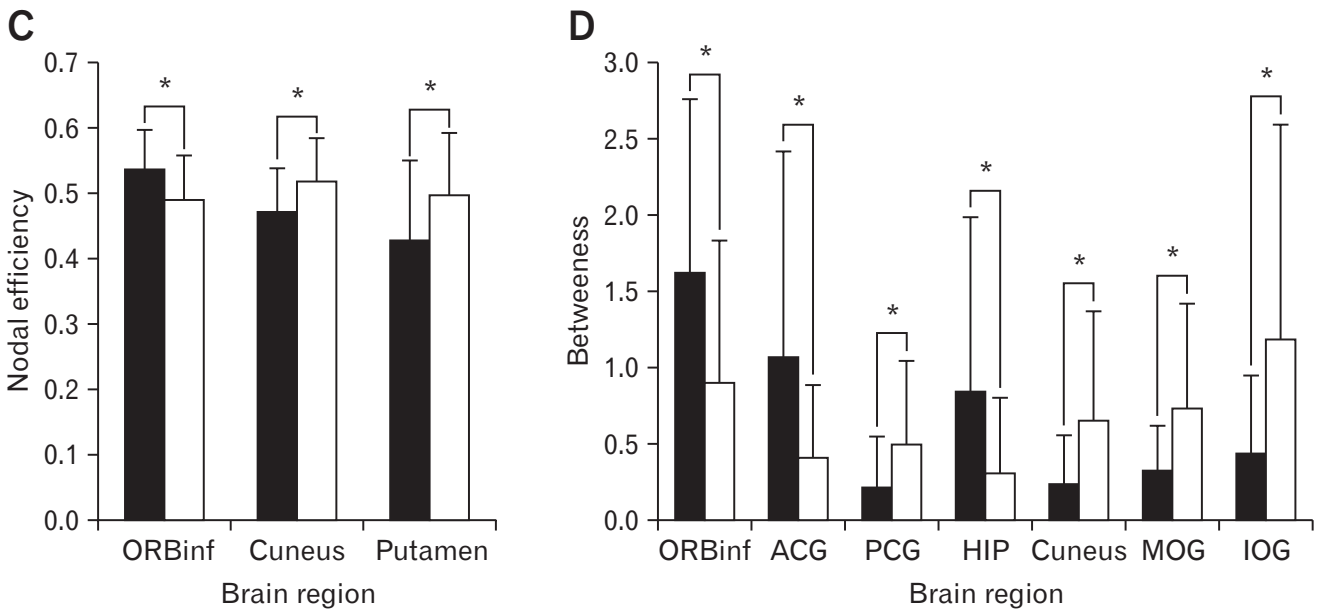

Figure 3. Brain areas that showed significant group differences. (A) Exhibited brain regions for at least 1 of the 3 nodal centrality measures. The bar graphs represent centrality metrics in patients and controls (black for patients; white for controls), including nodal degree (B), nodal efficiency (C), and betweeness (D). ACG, anterior cingulate gyrus; ORBinf, orbital part of the inferior frontal gyrus; HIP, hippocampus; PCG, posterior cingulate gyrus; MOG, middle occipital gyrus; IOG, inferior occipital gyrus; $\mathrm{FD}$, functional dyspepsia; $\mathrm{HC}$, healthy controls. ${ }^{*} P<0.05$, ${ }^{*} P<$ 0.01 .

A

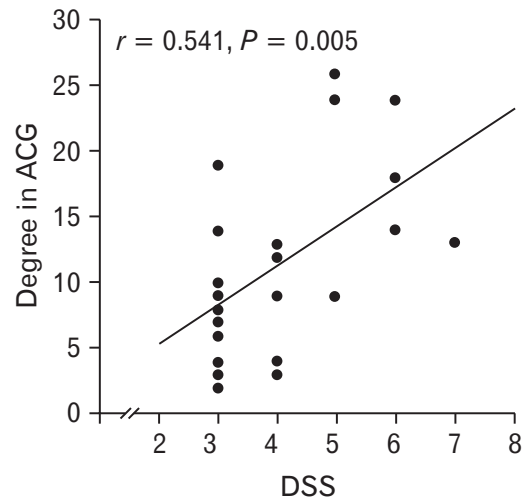

B

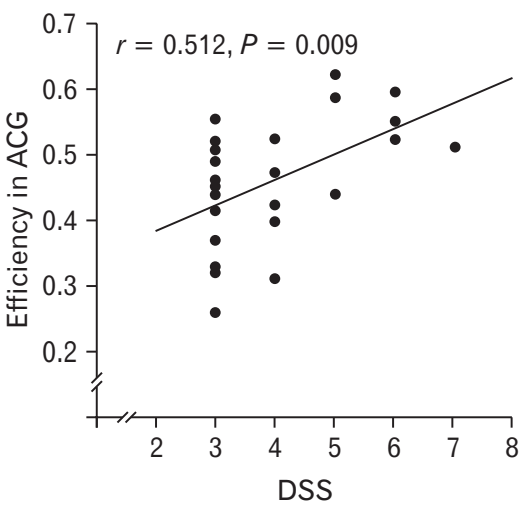

C

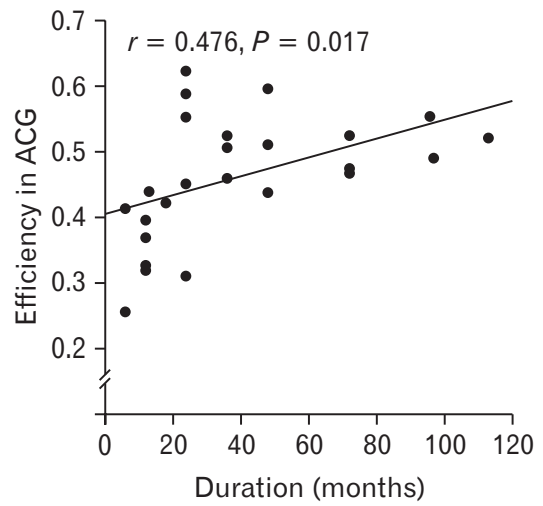

Figure 4. Correlation analysis between the nodal centrality in the anterior cingulate gyrus (ACG) and clinical variables for functional dyspepsia patients, including (A) the correlation between nodal degree and dyspepsia symptom score (DSS), (B) the correlation between nodal efficiency and DSS, and (C) the correlation between nodal efficiency and duration. 
Table 2. Overview of the Results for the Group Differences

\begin{tabular}{|c|c|c|c|c|c|c|}
\hline \multirow{2}{*}{ Parameters } & \multirow{2}{*}{ Node } & \multirow{2}{*}{ Hemisphere } & \multicolumn{2}{|c|}{ Group differences } & \multicolumn{2}{|c|}{$\begin{array}{c}\text { Group differences with SAS and } \\
\text { SDS as covariates }\end{array}$} \\
\hline & & & $\mathrm{T}^{\mathrm{a}}$ & $P$-value & $\mathrm{T}^{\mathrm{a}}$ & $P$-value \\
\hline $\mathrm{C}$ & - & - & 4.253 & $<0.001$ & 2.031 & 0.048 \\
\hline $\mathrm{L}$ & - & - & 1.999 & 0.051 & 1.880 & 0.066 \\
\hline $\mathrm{E}_{\text {local }}$ & - & - & 4.934 & $<0.001$ & 2.554 & 0.014 \\
\hline $\mathrm{E}_{\text {global }}$ & - & - & -0.847 & 0.401 & -0.452 & 0.653 \\
\hline \multirow[t]{2}{*}{$\mathrm{D}$} & ORBinf & $\mathrm{R}$ & 2.975 & 0.005 & 1.426 & 0.160 \\
\hline & Putamen & $\mathrm{R}$ & -2.183 & 0.034 & -2.952 & 0.005 \\
\hline \multirow[t]{3}{*}{$\mathrm{E}_{\text {nodal }}$} & ORBinf & $\mathrm{R}$ & 2.547 & 0.014 & 1.240 & 0.221 \\
\hline & Cuneus & $\mathrm{L}$ & -2.588 & 0.013 & -2.545 & 0.014 \\
\hline & Putamen & $\mathrm{R}$ & -2.206 & 0.032 & -3.47 & 0.001 \\
\hline \multirow[t]{7}{*}{ B } & ORBinf & $\mathrm{R}$ & 2.48 & 0.017 & 1.685 & 0.099 \\
\hline & $\mathrm{ACG}$ & $\mathrm{L}$ & 2.32 & 0.025 & 2.149 & 0.037 \\
\hline & HIP & $\mathrm{L}$ & 2.148 & 0.037 & 1.628 & 0.099 \\
\hline & PCG & $\mathrm{R}$ & -2.142 & 0.037 & -2.685 & 0.010 \\
\hline & Cuneus & $\mathrm{L}$ & -2.623 & 0.017 & -3.105 & 0.003 \\
\hline & MOG & $\mathrm{L}$ & -2.67 & 0.010 & -3.145 & 0.003 \\
\hline & $\mathrm{IOG}$ & $\mathrm{R}$ & -2.476 & 0.017 & -2.861 & 0.006 \\
\hline
\end{tabular}

${ }^{\mathrm{a}}$ Independent sample T-test.

SAS, self-rating anxiety scale; SDS, self-rating depression scale; C, clustering coefficient; L, shortest path length; $\mathrm{E}_{\text {global }}$, global efficiency; $\mathrm{E}_{\text {local }}$, local efficiency; D, nodal degree; $\mathrm{E}_{\text {nodal }}$, nodal efficiency; $\mathrm{B}$, nodal betweenness; ORBinf, orbital part of the inferior frontal gyrus; ACG, anterior cingulate gyrus; HIP, Hippocampus; PCG, posterior cingulate gyrus; MOG, middle occipital gyrus; IOG, inferior occipital gyrus; R, right; L, left.

symptoms.

Additionally, to detect potential influences of anxiety and depression on network attributes, group differences in network attributes were observed after controlling SAS and SDS scores. Results found that group differences remained significant for the clustering coefficient, local efficiency, and most nodal centralities after controlling the SAS and SDS scores, as shown in Table 2. Nevertheless, the inclusion of SAS and SDS scores as covariates erased the group differences in nodal centralities in the ORBinf and hippocampus. Of note, the above results could not withstand the false discovery rate (FDR) correction except for the group differences in the clustering coefficient and local efficiency before controlling for SAS and SDS. Moreover, we performed a partial correlation between the network metrics and DSS while controlling for SAS and SDS, and found that the correlation persisted (degree-DSS: $r=0.564$, $P=0.005$; nodal efficiency-DSS: $r=0.512, P=0.013$; nodal efficiency-duration: $r=0.047, P=0.023$ ).

\section{Discussion}

The current study investigated the topological properties of brain functional networks in FD patients involving a small-world model, network efficiency and nodal centralities. The results exhibited that FD patients had an increased clustering coefficient and local efficiency relative to controls. Moreover, several regions showed enhanced centralities in the right ORBinf, left ACG, and left hippocampus, and decreased centralities in the right $\mathrm{PCG}$, right putamen, left cuneus, left MOG, and right IOG. In particular, the centrality of the ACG was associated with the severity and duration of the illness. The above results provided a fresh perspective on our understanding of the pathophysiology of FD by revealing the link between network organization and FD.

In the current study, FD patients and controls showed smallworld characteristics in their intrinsic brain networks. Small-world topology represents an organization with high clustering and short path length, and thus a brain network with small-world attributes satisfies the processing demands of local clustering and global integration simultaneously. ${ }^{13}$ The existence of small-world properties in $\mathrm{FD}$ patients assures the effectiveness of information propagation and processing with small wiring costs in the whole-brain network. ${ }^{25}$ Despite being "small-world," FD patients showed a significantly enhanced clustering coefficient but no significant change in the shortest path length. Since the clustering coefficient reflects local specialization and shortest path length characterizes global integra- 
tion, our findings revealed that FD had a higher ability for specialized processing while maintaining the effectiveness of global integration. Moreover, we also found enhanced local efficiency in FD patients. Local efficiency, a function of wiring cost (path length), estimates the ability of the brain network to transfer parallel information within cliques. ${ }^{26}$ Thus, the enhancement of local efficiency reflects a lower cost of information transfer within clusters. The result further supported disrupted local functioning in $\mathrm{FD}$ patients, which may imply adaptive mechanisms for maintaining balance in the human body.

Nodal centrality detects the importance of brain nodes by measuring the information flow in the functional connectome. ${ }^{12}$ In our results, FD-related increases in nodal centralities were mainly found in the ORBinf (a part of the orbito-frontal cortex $[\mathrm{OFC}]$ ), ACG, and hippocampus. The OFC and ACG are the most prominent regions within the salience network - a system that integrates sensory data underlying interoceptive-autonomic processing. ${ }^{27}$ The OFC, a cortex on the ventral surface of the frontal lobe, engages in social adjustment, decision-making, reward learning, emotional processing, and self-monitoring. ${ }^{28-32}$ It is a nexus for integrating sensory information and modulating autonomic reactions, ${ }^{33}$ and can receive information from multiple sensory input. ${ }^{33,34}$ In our results, the inclusion of SAS and SDS as covariates erased the group differences in the ORBinf, which indicated that its centrality might be closely related to emotional factors integrated in visceral perception. The ACG is a multifunctional region that participates in information processing of visceral sensory data, cognitive control and emotional arousal. $^{35-38}$ It is one of the most commonly activated areas during visceral distension. ${ }^{39,40}$ Intriguingly, the nodal centralities in the ACG were found to be positively related to DSS of patients in the current results, which reflected the correlation between information flow through the ACG and symptom severity. To our knowledge, the symptom severity (subjective experience) is determined by multiple factors involving emotion, cognition, past experience, further prediction, and so on. ${ }^{3}$ Therefore, we suspected that the ACG may be closely linked to FD-related experiences through its relationship with the multifactorial integration in the brain network. This is to some extent in concordance with our previous finding that the ACG was related to the symptom severity of FD patients. ${ }^{9,41}$ The hippocampus is an important area associated with memory formation, organization and storage. Larsson found that normosensitive patients with IBS showed more activation in the hippocampus during expectation of visceral distention than controls. ${ }^{42}$ This indicated that impending visceral stimuli may spark memories of symptom perception. Moreover, the hippocampus has been implicated in the exacerbation of pain driven by anxiety. ${ }^{43}$ In the current study, the disappearance of aberrant centrality in the hippocampus after following SAS and SDS as covariates seems to validate the viewpoint. We speculated that abnormally increased centrality in the hippocampus may cause or increase perceptual sensitivity, probably due to past discomfort experience and/or increased anxiety/depression levels. Therefore, the strengthened effects of the above 3 regions are likely to be associated with emotion, memory, cognition as well as sensory.

In the current study, we also found an FD-related decrease in nodal centralities in the PCG, putamen, cuneus, MOG, and IOG. The PCG is a pivotal node of the default mode network ${ }^{44}$ and has been identified as a hub in functional connectivity. ${ }^{45}$ Its activity reflects conscious awareness, the arousal state and the focus of attention. ${ }^{46}$ Van Oudenhove et $\mathrm{al}^{47}$ reported deactivations in the PCG during gastric distension. ${ }^{47}$ They interpreted the deactivations as functional attenuation in the default mode network during interoceptive stimulation. ${ }^{47}$ However, we considered the decreased centrality in the PCG to be related to the focus and breadth of attention. Attentional regulation is an important factor of the etiology underlying heightened visceral pain. ${ }^{48}$ Attention to pain can result in an increase in pain intensity possibly through the alterations of descending nociceptive modulation. ${ }^{49}$ Therefore, it is possible that excessive attention to gastric pain or discomfort caused inhibition in the PCG ${ }^{50}$ The cuneus, MOG, and IOG are in the occipital lobe of the human brain involved in visual information processing. They have been reported to mediate direction-specified and selectionrelated attention. ${ }^{51,52}$ Wang et $\mathrm{al}^{53}$ examined the sensation threshold during rectal distention in IBS patients and found that distracting their attention by talking and reading markedly increased the threshold of sensation in these patients. ${ }^{53}$ This documented that selective attention of gastrointestinal symptoms is the cognitive-behavioral characteristic of IBS patients. The putamen is one important structure of the basal ganglia that is responsible for information integration between the thalamus and cortical regions. Previous brain imaging studies on FD reported higher glycometabolism in the basal ganglia ${ }^{41}$ and enhanced gray-matter volumes in the pumaten ${ }^{54}$ in patients compared with controls. Since the basal ganglia are commonly involved in pain processing including analgesia and pain modulation, ${ }^{55}$ the reduction of centrality in the putamen may represent inhibited pain modulation, contributing to gastric symptoms of FD patients. Accordingly, the reduction of centralities in the above regions might be the embodiment of inhibited pain modulation and selective attention to gastric sensation in $\mathrm{FD}$ patients.

In summary, we revealed a shift of the cortical network dur- 
ing resting-state in FD patients. However, the current study is a preliminary investigation for unweighted and undirected networks in FD patients based on 90 regions. According to previous reports, the weighted networks could yield crucial insights into the functional connectome by establishing a truer connectivity model for the human brain, ${ }^{56}$ whereas the directed networks may provide causal relationships between different brain areas. ${ }^{57}$ Moreover, the network topology may vary with a parcellation resolution. ${ }^{58}$ Therefore, further studies should clarify more topological properties based on a weighted and directed network at a different parcellation resolution and voxel-wise level.

\section{Conclusions}

The current study revealed topological alterations of the functional brain networks at the local and global level in FD patients, and the alterations may be associated with the synergistic effects of disturbances in emotion, memory, pain modulation, and selective attention in patients.

\section{Supplementary Materials}

Note: To access the supplementary tables mentioned in this article, visit the online version of Journal of Neurogastroenterology and Motility at http://www.jnmjournal.org/, and at http://dx.doi. org/10.5056/jnm15118.

Financial support: This work was supported by the Project for the National Natural Science Foundation of China under Grant Nos. $81501547,81571640,81471737,81501543,81371530,81501548$, 61374014 , and 81471738 .

\section{Conflicts of interest: None.}

Author contributions: Jiaofen Nan: processing data, interpreting data, and drafting the article; Li Zhang: processing data and interpreting data; Fubao Zhu and Xiaorui Tian: processing data; Qian Zheng: analyzing data; Karen M von Deneen: revising the manuscript; Jixin Liu: designing the study, collecting data, and interpreting data; and Ming Zhang: designing the study and collecting data.

\section{References}

1. Drossman DA, Dumitrascu DL. Rome III: new standard for functional gastrointestinal disorders. J Gastrointestin Liver Dis 2006;15:237-241.

2. Tack J, Masaoka T, Janssen P. Functional dyspepsia. Curr Opin Gastroenterol 2011;27:549-557.

3. Mayer EA, Gupta A, Kilpatrick LA, Hong JY. Imaging brain mechanisms in chronic visceral pain. Pain 2015;156(suppl 1):S50-S63.

4. Borsook D, Edwards R, Elman I, Becerra L, Levine J. Pain and analgesia: the value of salience circuits. Prog Neurobiol 2013;104:93-105.

5. Simons LE, Elman I, Borsook D. Psychological processing in chronic pain: a neural systems approach. Neurosci Biobehav Rev 2014;39:61-78.

6. Vandenberghe J, Dupont P, Van Oudenhove L, et al. Regional cerebral blood flow during gastric balloon distention in functional dyspepsia. Gastroenterology 2007;132:1684-1693.

7. Van Oudenhove L, Vandenberghe J, Dupont P, et al. Abnormal regional brain activity during rest and (anticipated) gastric distension in functional dyspepsia and the role of anxiety: a H215O-PET study. Am J Gastroenterol 2010;105:913-924.

8. Zeng F, Qin W, Liang F, et al. Abnormal resting brain activity in patients with functional dyspepsia is related to symptom severity. Gastroenterology 2011;141:499-506.

9. Nan J, Liu J, Zhang D, et al. Altered intrinsic regional activity and corresponding brain pathways reflect the symptom severity of functional dyspepsia. Neurogastroent Motil 2014;26:660-669.

10. Liu P, Zeng F, Zhou G, et al. Alterations of the default mode network in functional dyspepsia patients: a resting-state fmri study. Neurogastroenterol Motil 2013;25:e382-e388.

11. Nan J, Liu J, Li G, et al. Whole-brain functional connectivity identification of functional dyspepsia. PLos One 2013;8:e65870.

12. Rubinov M, Sporns O. Complex network measures of brain connectivity: uses and interpretations. Neuroimage 2010;52:1059-1069.

13. Sporns O. The human connectome: a complex network. Ann N Y Acad Sci 2011;1224:109-125.

14. Narr KL, Leaver AM. Connectome and schizophrenia. Curr Opin Psychiatry 2015;28:229-235.

15. Lei D, Li K, Li L, et al. Disrupted functional brain connectome in patients with posttraumatic stress disorder. Radiology 2015;276:818-827.

16. Zhang J, Wang J, Wu Q, et al. Disrupted brain connectivity networks in drug-naive, first-episode major depressive disorder. Biol Psychiatry 2011; 70:334-342.

17. Wang J, Qiu S, Xu Y, et al. Graph theoretical analysis reveals disrupted topological properties of whole brain functional networks in temporal lobe epilepsy. Clin Neurophysiol 2014;125:1744-1756.

18. Pedersen M, Omidvarnia AH, Walz JM, Jackson GD. Increased segregation of brain networks in focal epilepsy: an fMRI graph theory finding. Neuroimage Clin 2015;8:536-542.

19. Zung WW. A rating instrument for anxiety disorders. Psychosomatics 1971;12:371-379.

20. Zung WW, Richards CB, Short MJ. Self-rating depression scale in an outpatient clinic: further validation of the SDS. Arch Gen Psychiatry 1965;13:508-515.

21. Tzourio-Mazoyer N, Landeau B, Papathanassiou D, et al. Automated anatomical labeling of activations in SPM using a macroscopic anatomical parcellation of the MNI MRI single-subject brain. Neuroimage 
2002;15:273-289.

22. Wang J, Zuo X, He Y. Graph-based network analysis of resting-state functional MRI. Front Syst Neurosci 2010;4:16.

23. Zuo XN, Ehmke R, Mennes M, et al. Network centrality in the human functional connectome. Cereb Cortex 2012;22:1862-1875.

24. Liu J, Zhao L, Li G, et al. Hierarchical alteration of brain structural and functional networks in female migraine sufferers. PLoS One 2012;7: e5 1250.

25. Latora V, Marchiori M. Efficient behavior of small-world networks. Phys Rev Lett 2001;87:198701.

26. Achard S, Bullmore E. Efficiency and cost of economical brain functional networks. PLos Comput Biol 2007;3:e17.

27. Seeley WW, Menon V, Schatzberg AF, et al. Dissociable intrinsic connectivity networks for salience processing and executive control. J Neurosci 2007;27:2349-2356.

28. Cavada C, Schultz W. The mysterious orbitofrontal cortex. Foreword. Cereb Cortex 2000;10:205.

29. Beer JS, John OP, Scabini D, Knight RT. Orbitofrontal cortex and social behavior: integrating self-monitoring and emotion-cognition interactions. J Cogn Neurosci 2006;18:871-879.

30. Drevets WC. Neuroimaging and neuropathological studies of depression: implications for the cognitive-emotional features of mood disorders. Curr Opin Neurobiol 2001;11:240-249.

31. Rempel-CLower NL. Role of orbitofrontal cortex connections in emotion. Ann N Y Acad Sci 2007;1121:72-86.

32. Kahnt T, Chang LJ, Park SQ, Heinzle J, Haynes JD. Connectivitybased parcellation of the human orbitofrontal cortex. J Neurosci 2012;32: 6240-6250.

33. Kringelbach ML. The human orbitofrontal cortex: linking reward to hedonic experience. Nat Rev Neurosci 2005;6:691-702.

34. Price JL. Definition of the orbital cortex in relation to specific connections with limbic and visceral structures and other cortical regions. Ann N Y Acad Sci 2007;1121:54-71.

35. Beckmann M, Johansen-Berg H, Rushworth MF. Connectivity-based parcellation of human cingulate cortex and its relation to functional specialization. J Neurosci 2009;29:1175-1190.

36. Gao J, Wu X, Owyang C, Li Y. Enhanced responses of the anterior cingulate cortex neurones to colonic distension in viscerally hypersensitive rats. J Physiol 2006;570(Pt 1):169-183.

37. Yan N, Cao B, Xu J, Hao C, Zhang X, Li Y. Glutamatergic activation of anterior cingulate cortex mediates the affective component of visceral pain memory in rats. Neurobiol Learn Mem 2012;97:156-164.

38. Stevens FL, Hurley RA, Taber KH. Anterior cingulate cortex: unique role in cognition and emotion. J Neuropsychiatry Clin Neurosci 2011;23: 121-125.

39. Tillisch K, Mayer EA, Labus JS. Quantitative meta-analysis identifies brain regions activated during rectal distension in irritable bowel syndrome. Gastroenterology 2011;140:91-100.

40. Sheehan J, Gaman A, Vangel M, Kuo B. Pooled analysis of brain activity in irritable bowel syndrome and controls during rectal balloon distension. Neurogastroenterol Motil 2011;23:336-346, e158.

41. Nan J, Liu J, Mu J, et al. Brain-based correlations between psychological factors and functional dyspepsia. J Neurogastroenterol Motil
2015;21:103-110

42. Larsson MB, Tillisch $\mathrm{K}$, Craig AD, et al. Brain responses to expectation and delivery of a visceral stimulus in IBS reflect visceral sensitivity thresholds. Gastroenterology 2012;142:463-472, e3.

43. Ploghaus A, Narain C, Beckmann CF, et al. Exacerbation of pain by anxiety is associated with activity in a hippocampal network. J Neurosci 2001;21:9896-9903.

44. Fransson P, Marrelec G. The precuneus/posterior cingulate cortex plays a pivotal role in the default mode network: evidence from a partial correlation network analysis. Neuroimage 2008;42:1178-1184.

45. Tomasi D, Volkow ND. Association between functional connectivity hubs and brain networks. Cereb Cortex 2011;21:2003-2013.

46. Leech R, Sharp DJ. The role of the posterior cingulate cortex in cognition and disease. Brain 2014;137(Pt 1):12-32.

47. Van Oudenhove L, Vandenberghe J, Dupont P, et al. Cortical deactivations during gastric fundus distension in health: visceral pain-specific response or attenuation of 'default mode' brain function? A $\mathrm{H}_{2}{ }^{15} \mathrm{O}-\mathrm{PET}$ study. Neurogastroenterol Motil 2009;21:259-271.

48. Dunckley P, Aziz Q, Wise RG, Brooks J, Tracey I, Chang L. Attentional modulation of visceral and somatic pain. Neurogastroenterol Motil 2007; 19:569-577.

49. Bantick SJ, Wise RG, Ploghaus A, Clare S, Smith SM, Tracey I. Imaging how attention modulates pain in humans using functional MRI. Brain 2002;125(Pt 2):310-319.

50. Small DM, Gitelman DR, Gregory MD, Nobre AC, Parrish TB, Mesulam MM. The posterior cingulate and medial prefrontal cortex mediate the anticipatory allocation of spatial attention. Neuroimage 2003;18:633641.

51. Simpson GV, Weber DL, Dale CL, et al. Dynamic activation of frontal, parietal, and sensory regions underlying anticipatory visual spatial attention. J Neurosci 2011;31:13880-13889.

52. Tu S, Qiu J, Martens U, Zhang Q. Category-selective attention modulates unconscious processes in the middle occipital gyrus. Conscious Cogn 2013;22:479-485.

53. Wang W, Pan G, Qian J. Effect of psychological factors on visceral sensation of patients with irritable bowel syndrome. Zhonghua Yi Xue Za Zhi 2002;82:308-311.

54. Liu P, Zeng F, Yang F, et al. Altered structural covariance of the striatum in functional dyspepsia patients. Neurogastroent Motil 2014;26:11441154.

55. Schweinhardt P, Kuchinad A, Pukall CF, Bushnell MC. Increased gray matter density in young women with chronic vulvar pain. Pain 2008;140: 411-419.

56. Barrat A, Barthélemy M, Pastor-Satorras R, Vespignani A. The architecture of complex weighted networks. Proc Natl Acad Sci USA 2004;101: 3747-3752.

57. Liao W, Ding J, Marinazzo D, et al. Small-world directed networks in the human brain: multivariate Granger causality analysis of resting-state fMRI. Neuroimage 2011;54:2683-2694.

58. Fornito A, Zalesky A, Bullmore ET. Network scaling effects in graph analytic studies of human resting-state FMRI data. Front Syst Neurosci 2010;4:22. 